\title{
Erdheim-Chester Disease Presenting with Pneumothorax
}

\author{
Masafumi Yamaguchi ${ }^{a}$ b Tetsuhiro Shiota $^{a}$ Yoichiro Kobashi $^{c}$ \\ ${ }^{a}$ Division of Respiratory Medicine, Ako City Hospital, Ako, ${ }^{b}$ Department of Cardiovascular and Respiratory \\ Medicine, Shiga University of Medical Science, Otsu, and ' ${ }^{\mathrm{D}}$ Department of Pathology, Tenri Hospital, Tenri, Japan
}

\section{Established Facts}

- Erdheim-Chester disease may present with pulmonary involvement leading to chronically progressive pulmonary symptoms.

\section{Novel Insights}

- Erdheim-Chester disease may present with acute pulmonary symptoms due to rupture of a large cystic lesion with resultant pneumothorax. Clinicians must be aware that patients with Erdheim-Chester disease may exhibit a wide range of clinical manifestations.

\section{Key Words}

Erdheim-Chester disease $\cdot$ Pneumothorax $\cdot$ Histiocytosis

\begin{abstract}
Erdheim-Chester disease (ECD) is a rare non-Langerhans cell histiocytosis most commonly characterized by symmetrical skeletal involvement and may present with pulmonary involvement leading to chronically progressive pulmonary symptoms. Characteristics on chest radiography include non-specific findings of diffuse interstitial and pleural thickening, micronodules, ground-glass opacities and parenchymal condensation as a result of infiltration by lipid-laden histiocytes. We present the case of a 50-year-old man with ECD
\end{abstract}

presenting with acute pulmonary symptoms due to rupture of a large cystic lesion with resultant pneumothorax. He was brought by ambulance to our hospital, complaining of acute anterior chest pain and severe dyspnea. Chest radiography showed right-sided pneumothorax with a collapsed lung, a large, left-sided cystic lesion in the upper lung field and accentuated interstitial markings. Bullectomy and surgical biopsy were performed, demonstrating histologically histiocytic infiltrates that were strongly positive for CD68, but negative for S-100 protein and CD1a. Subsequent systemic examinations indicated widespread symmetrical skeletal involvement, leading to a definitive diagnosis of ECD.

Copyright $\odot 2011$ S. Karger AG, Basel

\section{KARGER}

Fax +4161306 1234

E-Mail karger@karger.ch

www.karger.com
(C) 2011 S. Karger AG, Basel

$0025-7931 / 11 / 0826-0552 \$ 38.00 / 0$

Accessible online at:

www.karger.com/res
Masafumi Yamaguchi, MD, $\mathrm{PhD}$

Department of Cardiovascular and Respiratory Medicine, Shiga University of Medical Science, Seta Tsukinowa-cho

Otsu, Shiga 520-2192 (Japan)

Tel.+81 77548 2213, E-Mail myama3265@gmail.com 

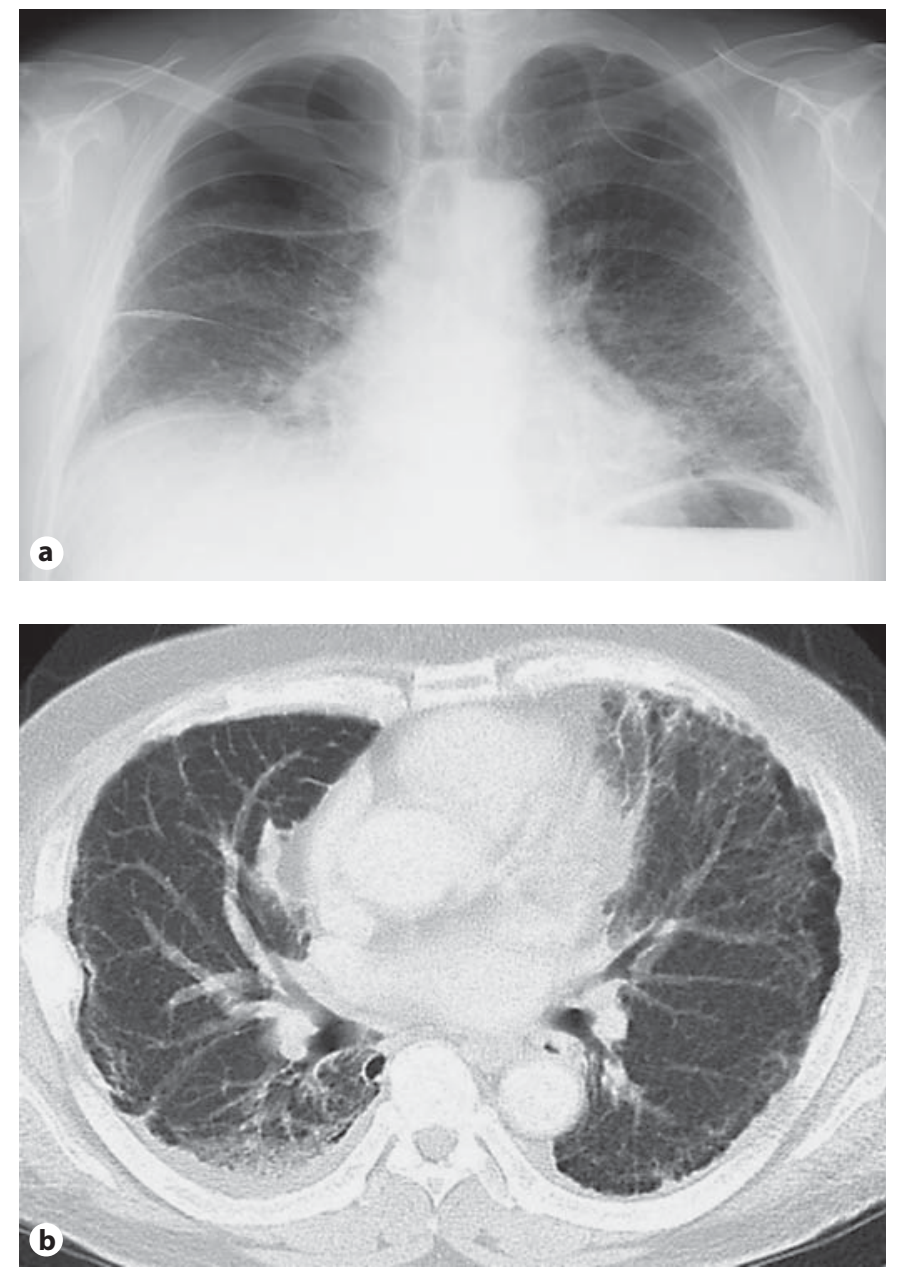

\section{Introduction}

Erdheim-Chester disease (ECD) was initially described in 1930 by William Chester as a non-Langerhans cell histiocytosis of unknown cause [1]. This disorder is characterized by symmetric bilateral osteosclerosis of long tubular bones. Almost all patients display extraskeletal manifestations, and about $40 \%$ experience pulmonary manifestations [2]. ECD is a progressive disease [3] despite the availability of a wide variety of treatments, including corticosteroids, radiotherapy, immunotherapy and chemotherapy.

The major pulmonary features of ECD on radiography include smooth interlobular septal thickening, micronodules, ground-glass opacities, thickening of interlobular fissures and parenchymal condensation, which results from infiltration of lipid-laden histiocytes $[2,4]$. Cystic airspaces of small size caused by architectural de-

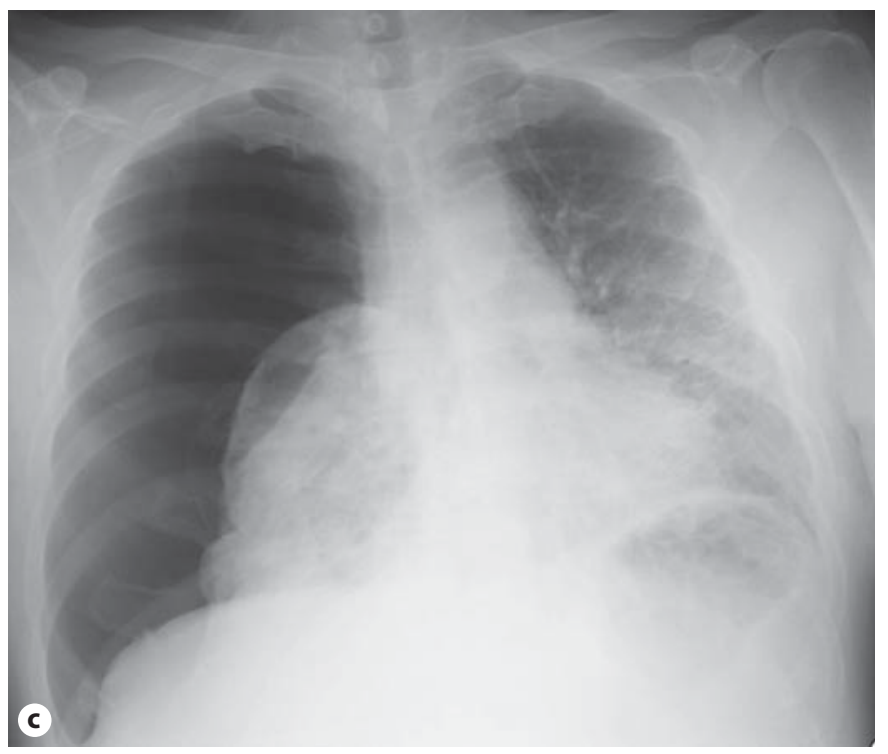

Fig. 1. Chest radiography (a) and CT (b) about 1 year before admission, showing cystic lesions in bilateral upper lung fields, diffuse pleural thickening and effusions, interlobular septal thickening and patchy areas of ground-glass opacity. c Chest radiography on presentation to the emergency department, showing pneumothorax with a collapsed lung on the right side, and a large cystic lesion in the upper lung field and accentuated interstitial markings on the left side.

struction in the fibrotic lung are also rarely identified [4], although large cystic lesions have not previously been demonstrated. Immunohistochemically, histiocytes in ECD are positive for CD68 and negative for CDla, with variable staining for S-100 protein [5].

We present herein the case of a 50 -year-old man with ECD detected following acute anterior chest pain and severe dyspnea without any skeletal symptoms, and identification of pneumothorax, a large cystic lesion and accentuated interstitial markings on chest radiography.

\section{Case Report}

A 50-year-old Japanese man was admitted to the emergency department of our hospital by ambulance, complaining of acute anterior chest pain and severe dyspnea. He was an ex-smoker of 25 pack-years (having quit smoking 5 years ago). He had been asymptomatic until 6 months before admission, at which time he 


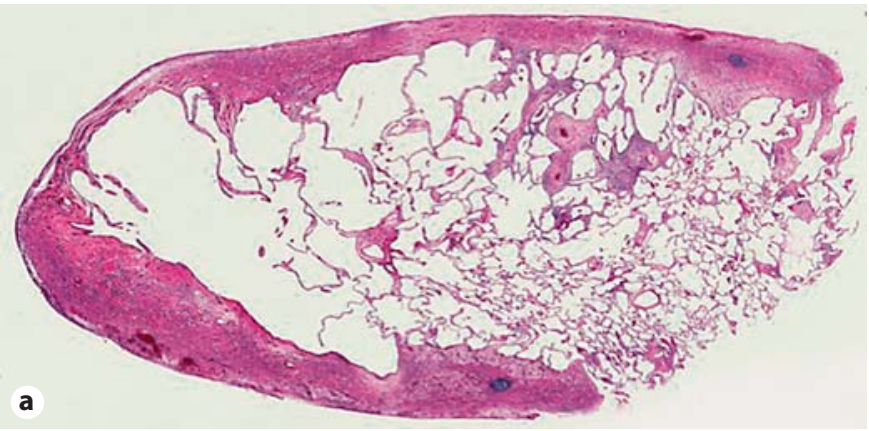

Fig. 2. a Lung biopsies of the right lower lung field revealing marked thickening of visceral pleura and interstitial fibrosis, and emphysematous changes mainly in subpleural areas. b Histiocytes in the thickened area of visceral pleura showing positive staining for CD68.

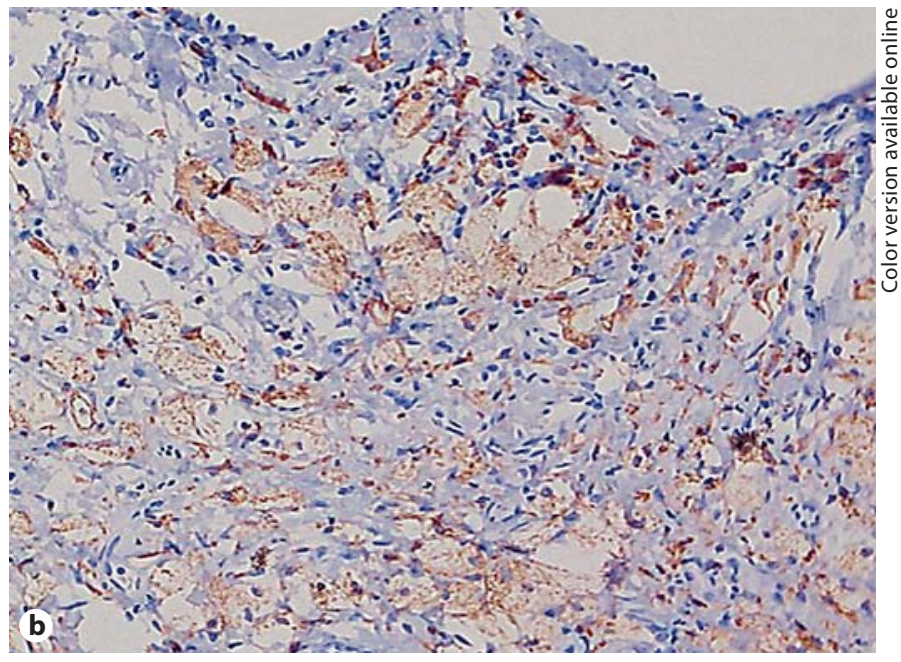

(MRI) (fig. 3a, b). Abdominal CT, cardiac echography and brain MRI revealed normal findings. A definitive diagnosis of ECD was confirmed on the basis of combined clinical, radiological and pathological findings.

The patient is now being treated with systemic prednisolone. However, he has demonstrated gradual symptomatic and functional deterioration, and chest radiography has shown increased bilateral interstitial fibrosis and enlargement of the left-sided cystic lesion. Cyclophosphamide was discontinued only 1 week after initiation due to severe nausea and consequent loss of appetite. blood gas analysis showed a $\mathrm{pH}$ of 7.400 , with $\mathrm{PaO}_{2}$ at $62.5 \mathrm{~mm}$ $\mathrm{Hg}$ and $\mathrm{PCO}_{2}$ at $35.4 \mathrm{~mm} \mathrm{Hg}$, while the patient was breathing oxygen at a rate of $5 \mathrm{l} / \mathrm{min}$ by mask. Chest radiography demonstrated right-sided pneumothorax with a collapsed lung, left-sided large cystic lesion in the upper lung field and accentuated interstitial markings (fig. 1c). Results of laboratory testing performed on admission were within normal ranges.

The disease was treated immediately using a chest tube drain, but air leakage continued and expansion of subcutaneous emphysema was induced. Six days after admission, the patient underwent bullectomy in the form of thoracotomy. Informed consent was obtained preoperatively from the patient for the purpose of further examination of pulmonary involvement, and a surgical lung biopsy was additionally performed from the right lower lobe. The postoperative course was uneventful and the patient made a smooth recovery. He was discharged 8 days after surgery.

Histological findings of the right lower lobe indicated marked thickening of the visceral pleura and interstitial fibrosis, as well as emphysematous changes mainly in subpleural areas (fig. 2a). These abnormalities were expanded chiefly in interlobular septa and bronchovascular bundles. The area of thickened visceral pleura was accompanied by a combination of fibrosis and lymphocyte infiltration, which were characteristic in that a cluster of large histiocytes was seen, most of which were positive for CD68 (fig. 2b), but negative for S-100 and CD1a.

Although the patient was free of skeletal symptoms, we confirmed skeletal involvement of bilateral distal radii, ulnae, femora and tibiae on bone scintigraphy and magnetic resonance imaging

\section{Discussion}

ECD is a rare non-Langerhans cell histiocytosis that may present with progressive pulmonary symptoms. The most common manifestation at presentation is symmetrical long bone pain with associated osteosclerotic lesions, whereas ECD presenting with non-specific respiratory symptoms as a sole manifestation is uncommon [69]. Accordingly, differential diagnosis in such cases may delay correct diagnosis of ECD [10]. The present patient was distinctive in that he demonstrated only acute symptoms of pulmonary involvement.

The most common pulmonary findings for ECD on radiography include smooth interlobular septal thickening, micronodules, ground-glass opacities, thickening of interlobular fissures and parenchymal condensation, which results from infiltration of lipid-laden histiocytes $[2,4]$. Cystic airspaces of small size caused by architectural destruction are also rarely identified [4]. However, no case reports of ECD have previously described large 


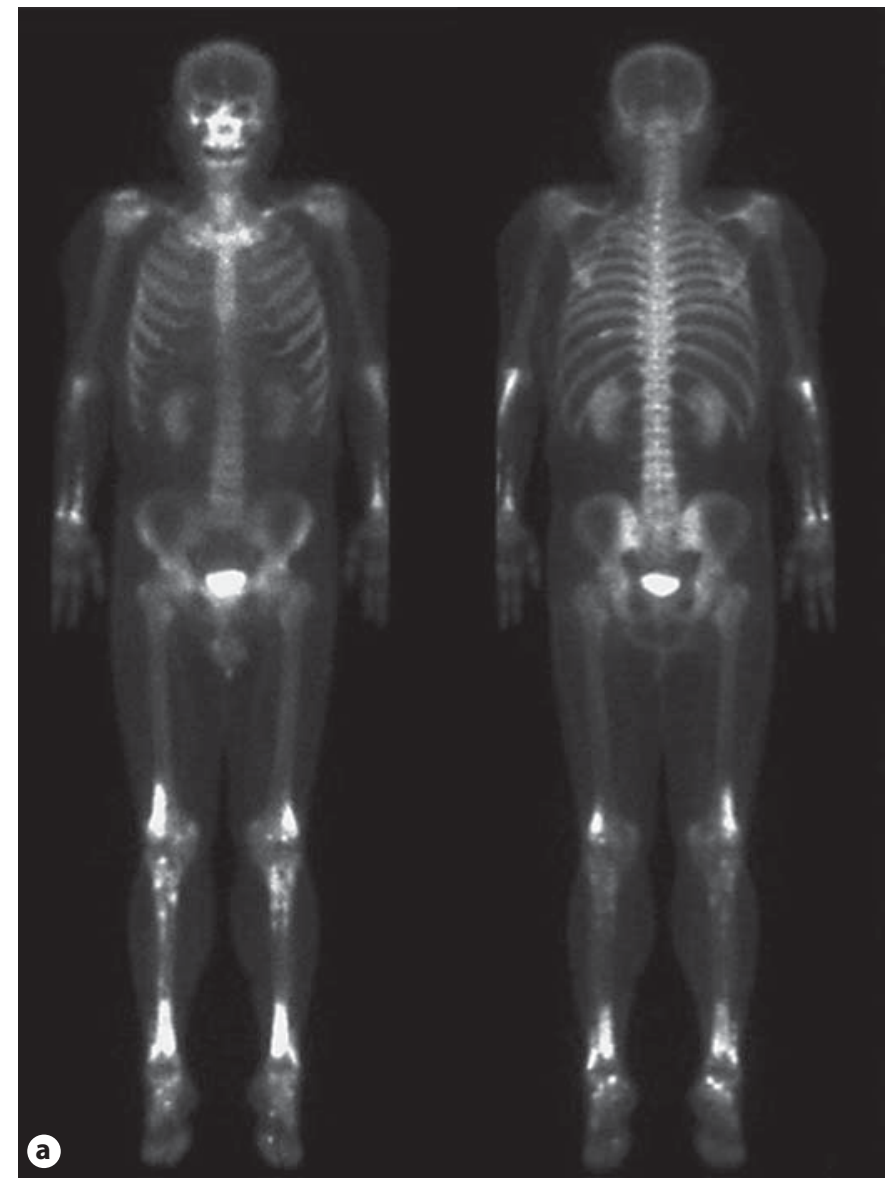

cystic lesions of the lung or pneumothorax induced by lesion rupture, although smoking-related emphysema may have been responsible for pneumothorax in this case, given that emphysematous changes were demonstrated in subpleural areas. Preoperative differential diagnoses of radiological characteristics include sarcoidosis, amyloidosis, alveolar proteinosis and other causes of interstitial thickening. Preoperative diagnosis of ECD can thus prove difficult when based only on clinical and radiological pulmonary manifestations.

Rush et al. [5] indicated that histiocytes in ECD were immunophenotypically positive for CD68 and negative for CD1a, and also showed variable staining with S-100 protein. Histopathological features in our patient included accumulation of foamy histiocytes along the thickening pleura and interlobular septa that showed staining for CD68, but not for S-100 or CD1a. Skeletal involvement was subsequently documented despite the lack of skeletal symptoms. The proper diagnosis of ECD was ascertained only after surgical biopsy.

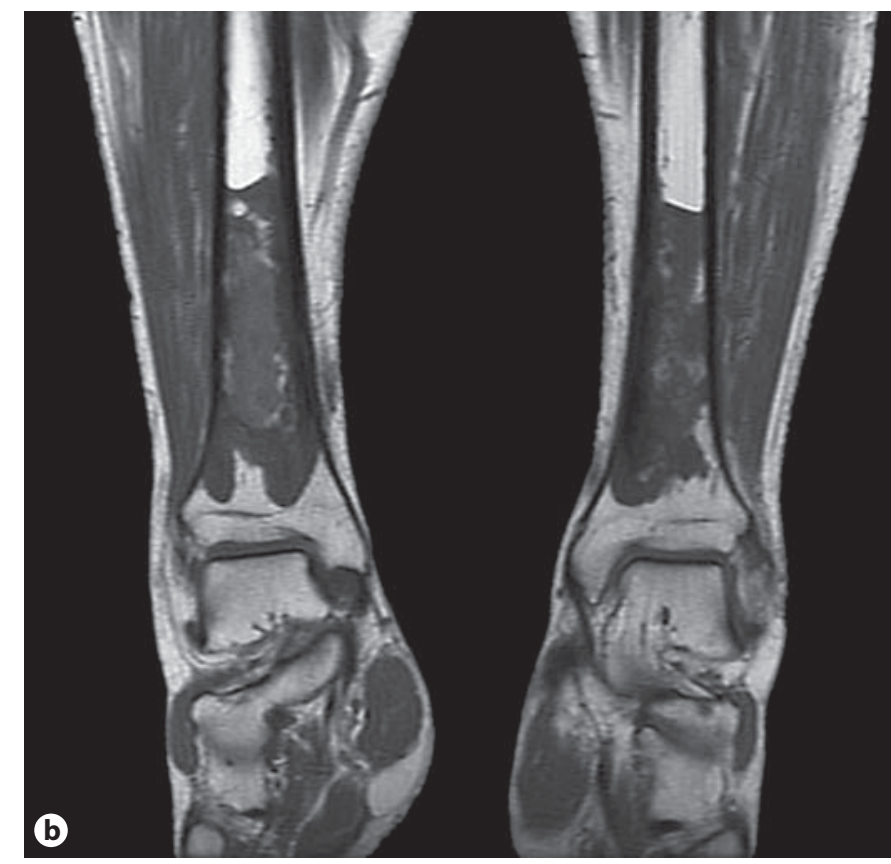

Fig. 3. a Bone scintigraphy revealing abnormally increased uptake bilaterally in the distal radii, ulnae, femora and tibiae. $\mathbf{b} \mathrm{T}_{1^{-}}$ weighted MRI demonstrating low signal intensity in metaphyseal regions of bilateral distal tibiae.

Sporadic reports have described glucocorticoids, cyclophosphamide, vincristine, adriamycin, colchicine and radiotherapy as effective against ECD due to the low frequency of this disorder $[6,7,11-13]$. In our patient, the first-choice treatment was systemic corticosteroids. However, little improvement was achieved and cyclophosphamide was unavailable because of severe side effects. Treatment with interferon- $\alpha$ will be considered now that patients treated with interferon- $\alpha$ have shown improved survival compared to those not receiving such treatment [2].

In summary, we present a case in which ECD was detected by acute pulmonary symptoms without any skeletal symptoms, due to rupture of a large cystic lesion with resultant pneumothorax. Clinicians must be aware that patients with ECD may exhibit a wide range of clinical manifestations. 


\section{References}

1 Chester W: Über Lipoidgranulomatose. Virchows Arch A Pathol Anat Histopathol 1930; 279:561-602.

-2 Arnaud L, Hervier B, Néel A, Hamidou MA, Kahn JE, Wechsler B, Pérez-Pastor G, Blomberg B, Fuzibet JG, Dubourguet F, Marinho A, Magnette C, Noel V, Pavic M, Casper J, Beucher AB, Costedoat-Chalumeau N, Aaron L, Salvatierra J, Graux C, Cacoub P, Delcey V, Dechant C, Bindi P, Herbaut C, Graziani G, Amoura Z, Haroche J: CNS involvement and treatment with interferon-alpha are independent prognostic factors in Erdheim-Chester disease: a multicenter survival analysis of 53 patients. Blood 2011;117:27782782.

3 Protopapadakis C, Antoniou KM, Nicholson AG, Voloudaki A, Tzanakis N, Karantanas A, Siafakas NM: Erdheim-Chester disease: pulmonary presentation in a case with advanced systemic involvement. Respiration 2009;77:337-340.
4 Brun AL, Touitou-Gottenberg D, Haroche J, Toledano D, Cluzel P, Beigelman-Aubry C, Piette JC, Amoura Z, Grenier PA: ErdheimChester disease: CT findings of thoracic involvement. Eur Radiol 2010;20:2579-2587.

$\rightarrow 5$ Rush WL, Andriko JA, Galateau-Salle F, Brambilla E, Brambilla C, Ziany-Bey I, Rosado-de-Christenson ML, Travis WD: Pulmonary pathology of Erdheim-Chester disease. Mod Pathol 2000;13:747-754.

6 6 Veyssier-Belot C, Cacoub P, Caparros-Lefebvre D, et al: Erdheim-Chester disease: clinical and radiologic characteristics of 59 cases. Medicine 1996;75:157-169.

-7 Egan AJM, Boardman LA, Tazelaar HD, et al: Erdheim-Chester disease: clinical, radiologic, and histopathologic findings in five patients with interstitial lung disease. Am J Surg Parthol 1999;23:17-26.

-8 Shamburek RD, Brewer HB, Gochuico BR: Erdheim-Chester disease: a rare multisystem histiocytic disorder associated with interstitial lung disease. Am J Med Sci 2001; 321:66-75.

-9 Allen TC, Chevez-Barrios P, Shetlar DJ, et al: Pulmonary and ophthalmic involvement with Erdheim-Chester disease: a case report and review of the literature. Arch Pathol Lab Med 2004;128:1428-1431.
10 Arnaud L, Pierre I, Beigelman-Aubry C, Capron F, Brun AL, Rigolet A, Girerd X, Weber N, Piette JC, Grenier PA, Amoura Z, Haroche J: Pulmonary involvement in Erdheim-Chester disease: a single-centre study of thirty-four patients and a review of the literature. Arthritis Rheum 2010;62:35043512.

11 Chung JH, Park MS, Shin DH, Choe KO, Kim SK, Chang J, Kim SK, Kim YS: Pulmonary involvement in Erdheim-Chester disease. Respirology 2005;10:389-392.

-12 Saboerali MD, Koolen MG, Noorduyn LA, van Delden OM, Bogaard HJ: Pleural thickening in a construction worker: it is not always mesothelioma. Neth J Med 2006;64: 88-90.

13 Loddenkemper K, Hoyer B, Loddenkemper C, Hermann KG, Rogalla P, Förster G, Buttgereit F, Hiepe F, Burmester GR: A case of Erdheim-Chester disease initially mistaken for Ormond's disease. Nat Clin Pract Rheumatol 2008;4:50-55. 